\title{
Impact of Malignancy Category on Vancomycin Dosage Requirements in Pediatric Oncology Patients
}

\section{Kyle A Franco* and Tara Higgins}

Department of Pharmacy, University of Florida Health Shands Hospital, USA

*Corresponding author: Kyle A Franco, Department of Pharmacy, University of Florida Health Shands Hospital, 231 East Chestnut St Louisville, KY 40202, USA, Tel: (502) 629-6000; Fax: (352) 265-1083; E-mail: kyleamberf@gmail.com

Received date: August 21, 2017; Accepted date: September 04, 2017; Published date: September 11, 2017

Copyright: ( 2017 Franco KA, et al. This is an open-access article distributed under the terms of the Creative Commons Attribution License, which permits unrestricted use, distribution, and reproduction in any medium, provided the original author and source are credited.

\begin{abstract}
Objectives: To determine the dosage of vancomycin in milligrams/kilogram/day (mg/kg/day) required to achieve a trough value of 10 to 20 micrograms/milliliter $(\mathrm{mcg} / \mathrm{mL})$ in pediatric patients with differing types of malignancies.

Methods: Retrospective review of pediatric patients with hematologic malignancy, solid tumor, or status post autologous or allogeneic hematopoietic stem cell transplantation (HSCT) receiving at least two doses of intravenous vancomycin with at least one evaluable trough concentration. Primary outcome was the dosage of vancomycin required to achieve first therapeutic trough value. Secondary endpoints included dosage requirement by age, proportion of patients achieving goal, incidence of supratherapeutic trough values and nephrotoxicity.
\end{abstract}

Results: Mean dosage requirements were $72.5[ \pm 2.3] \mathrm{mg} / \mathrm{kg} / \mathrm{day}$ among patients with hematologic malignancies, $66.5[ \pm 3.3] \mathrm{mg} / \mathrm{kg} /$ day in patients with solid tumors, and $77.3[ \pm 4.1] \mathrm{mg} / \mathrm{kg} /$ day in HSCT patients $(\mathrm{p}=0.12)$. Younger patients required significantly increased daily dosages in order to meet their trough goals in the hematologic malignancy and solid tumor groups $(p<0.05)$. The proportion of patients achieving trough goals was also similar between groups $(p=0.5)$. Supratherapeutic trough values were more common in the solid tumor groups $(p<0.05)$. Nephrotoxicity occurred more frequently in the HSCT group $(p<0.05)$.

Conclusion: Vancomycin dosage requirements are similar between pediatric patients with differing categories of malignancies. Patients with solid tumors and HSCT appear to be at higher risk for supratherapeutic trough values, and HSCT patients appear to be at an increased risk for nephrotoxicity.

Keywords: Pediatric malignancy; Pharmacokinetics; Vancomycin

\section{Abbreviations:}

Heme: Hematologic Malignancy; HSCT: Hematopoetic Stem Cell Transplant; ALL: Acute Lymphoblastic Leukemia; AML: Acute Myeloid Leukemia; CNS: Central Nervous System; Scr: Serum Creatinine.

\section{Introduction}

Pediatric patients with malignancy are immunocompromised by their disease state and its treatment. Their vulnerability to infection, propagated by frequent exposure to health care environments, central venous access devices, and compromised skin and mucous barriers, necessitates aggressive treatment at the first sign of infection. Vancomycin is a glycopeptide antibiotic relied upon for its activity against gram positive pathogens including methicillin-resistant staphylococcus aureus (MRSA) [1]. In pediatric patients with infections caused by this organism, a vancomycin dosage of 60 milligrams per kilogram per day $(\mathrm{mg} / \mathrm{kg} /$ day) is recommended, with therapeutic monitoring targeting a minimum concentration $\left(\mathrm{C}_{\min }\right)$ or trough value in the range of 15 to 20 micrograms per milliliter $(\mathrm{mcg} / \mathrm{mL})$ [2]. Optimal vancomycin trough concentrations for treatment of febrile neutropenia have not been well established, however ranges between 10 to $20 \mathrm{mcg} / \mathrm{mL}$ have been suggested based on a target area under the curve (AUC) to minimum inhibitory concentration (MIC) ratio of greater than $400[3,4]$. A prospective comparison of 30 children with malignancy being treated for neutropenic fever and 8 otherwise healthy children being treated for MRSA bacteremia found that while the two groups had similar maximum concentrations $\left(\mathrm{C}_{\max }\right)$ at steady state, the mean minimum concentrations $\left(\mathrm{C}_{\mathrm{min}}\right)$ were significantly lower among the malignancy group despite receiving the same total daily dosage. Patients with malignancy also demonstrated increased vancomycin clearance and shorter half-lives than their control counterparts, suggesting that in the presence of malignancy, vancomycin pharmacokinetics are altered [5]. These findings are supported by pharmacokinetic modelling and Monte Carlo simulation based upon 98 vancomycin concentrations from 70 children with hematologic malignancies receiving dosages of 40 to $60 \mathrm{mg} / \mathrm{kg} / \mathrm{day}$. This analysis found only $15 \%$ of infants and $24 \%$ of children reached AUC to MIC values above 400. A dosage regimen of $90 \mathrm{mg} / \mathrm{kg} /$ day in infants and $80 \mathrm{mg} / \mathrm{kg} /$ day in children was predicted to achieve target values in $50 \%$ of patients with a $6 \%$ increased risk of supratherapeutic troughs [6]. Similar results were reported in a retrospective review of vancomycin serum trough concentrations in 124 hospitalized pediatric oncology patients receiving $60 \mathrm{mg} / \mathrm{kg} /$ day reported a mean trough value of $7.1 \mathrm{mcg} / \mathrm{mL}$. After dosage adjustment patients less than 12 years of age required an average of $91 \mathrm{mg} / \mathrm{kg} / \mathrm{day}$ to achieve a trough concentration of $8-15 \mathrm{mcg} / \mathrm{mL}$. Trough concentrations were found to correlate with age, weight, and serum creatinine. An evaluation of underlying diagnosis was not conducted, 
although $42 \%$ of patients were noted to have hematologic malignancy and $58 \%$ solid tumors [7]. The impact of malignancy category on vancomycin dosage requirements in pediatric patients remains unknown and warrants further investigation.

\section{Methods}

A single center retrospective chart review was conducted to evaluate vancomycin dosage requirements among pediatric oncology patients. Patients cared for by the pediatric haematology/oncology/HSCT service between June 12011 and June 302015 were screened for inclusion. Patients were eligible for inclusion if less than or equal to 18 years of age, receiving treatment for malignancy or within 100 days from autologous or allogeneic HSCT, who received at least two doses of intravenous vancomycin with at least one evaluable trough concentration. Trough values were considered evaluable if drawn within one hour of the true trough. Patients were excluded if they received hematopoietic stem cell transplant for a non-oncologic indication, if they received renal replacement therapy, or had an estimated creatinine clearance less than 50 milliliters/minute $/ 1.73 \mathrm{~m}^{2}$ $\left(\mathrm{mL} / \mathrm{min} / 1.73 \mathrm{~m}^{2}\right)$ during vancomycin treatment. Creatinine clearance was estimated by the bedside schwartz equation.

\section{Endpoints}

The primary objective was to determine the mean dosage of vancomycin $(\mathrm{mg} / \mathrm{kg} /$ day $)$ required to achieve the first therapeutic trough value of 10 to $20 \mathrm{mcg} / \mathrm{mL}$ in pediatric patients within the following three groups: patients with solid tumors, patients with hematologic malignancies, and patients receiving HSCT. Secondary endpoints included the mean dosage requirement $(\mathrm{mg} / \mathrm{kg} /$ day $)$ required to attain therapeutic trough values among patients in the following age groups: $<1$ year, 1 to 5 years, 6 to 11 years, and $>11$ years of age. The percent difference between starting dose and therapeutic dose was evaluated along with the proportion of patients achieving a goal trough value, the days to therapeutic dosage, and days of vancomycin treatment. Safety outcomes assessed the incidence of supratherapeutic trough values and nephrotoxicity, which was defined as a greater than $50 \%$ increase in baseline serum creatinine or a greater than 0.3 milligrams/decilitre $(\mathrm{mg} / \mathrm{dL})$ increase in serum creatinine within a 48 hour timeframe.

\section{Statistical Analysis}

Patient demographics were evaluated using descriptive statistics. Primary and secondary outcomes that were normally distributed continuous variables were analyzed using analysis of variance (ANOVA) or repeated measures ANOVA. All categorical data outcomes were analyzed with Chi Square or fisher's exact testing as appropriate. This study was approved by the University of Florida Institutional Review Board (IRB) and informed consent was not required.

\section{Results}

A total of 262 vancomycin courses from 162 patients were screened for inclusion into the trial, and 104 courses were excluded for the reasons outlined in Figure 1 leaving 88 courses in the hematologic malignancy group, 44 in the solid tumor group, and 26 in the HSCT group.

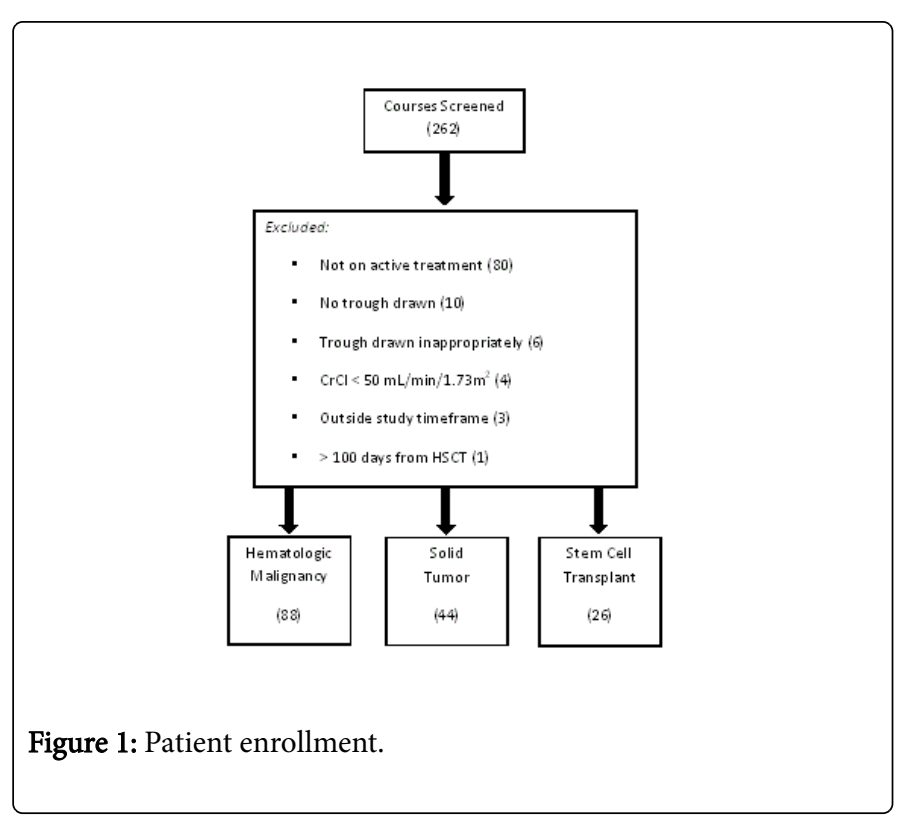

There were no significant differences among the baseline characteristics of patients in each group (Table 1). The hematologic malignancy group included 49 patients with Acute Myeloid Leukemia (21\%). Hematopoietic stem cell transplants were performed more often in patients with solid tumors $(73 \%)$ than those with hematologic malignancies (27\%). While only $46 \%$ of patients demonstrated positive cultures, the majority of positive cultures did yield an organism susceptible to vancomycin therapy. The mean dosage requirement required to attain the first therapeutic trough value between 10 and 20 $\mathrm{mcg} / \mathrm{mL}$ was $72.5[ \pm 2.3] \mathrm{mg} / \mathrm{kg} /$ day among patients with hematologic malignancies, $66.5[ \pm 3.3] \mathrm{mg} / \mathrm{kg} /$ day in patients with solid tumors, and $77.3[ \pm 4.1] \mathrm{mg} / \mathrm{kg} /$ day in patients receiving a hematopoietic stem cell transplant $(\mathrm{p}=0.12)$. Patients who never achieved this goal were excluded from the primary endpoint analysis. A sub-analysis of dosage requirements by age group revealed that within the hematologic malignancy and solid tumor groups, younger patients required increased daily dosages in order to meet their trough goals (Table 2).

This difference was statistically significant among patients with hematologic malignancies $(\mathrm{p}<0.05)$ and solid tumors $(\mathrm{p}<0.05)$. The mean difference between the starting and therapeutic dosage was not different between groups $(\mathrm{p}=0.87)$. The proportion of patients that achieved their target trough was also similar between groups $(\mathrm{p}=0.5)$, as was the mean number of dose changes ( $p=0.7$ ), days to therapeutic range $(\mathrm{p}=0.6)$, and mean days of treatment $(\mathrm{p}=0.5)$ (Table 3$)$. Supratherapeutic trough values were observed more often in the solid tumor group and the HSCT group than the hematologic malignancy group, respectively ( $38.6 \%$ vs. $34.6 \%$ vs. $18.2 \%$, p $<0.05)$.

Nephrotoxicity occurred more frequently in the hematopoietic stem cell transplant group than both the hematologic malignancy group and the solid tumor group, correspondingly $(23.1 \%$ vs. $3.5 \%$ vs. $0 \%$, $\mathrm{p}<0.05)$.Notably it was found that $81 \%$ of patients in the hematopoietic stem cell transplant patients were on concomitant nephrotoxins during their vancomycin therapy, compared with $63 \%$ of patients in the hematologic malignancy group and $52 \%$ in the solid tumor group. 
Citation: Franco KA, Higgins T (2017) Impact of Malignancy Category on Vancomycin Dosage Requirements in Pediatric Oncology Patients. J Pharmacovigil 5: 238. doi:10.4172/2329-6887.1000238

Page 3 of 5

\begin{tabular}{|c|c|c|c|}
\hline \multirow{2}{*}{ Parameter } & Heme & Solid Tumor & HSCT \\
\hline & $(n=88)$ & $(n=44)$ & $(n=26)$ \\
\hline Median age (years) [IQR] & 5 [2-11] & $8.5(3-14)$ & $7.08(3-13)$ \\
\hline Male, n (\%) & $44(50)$ & $19(43.2)$ & $15(57.7)$ \\
\hline Median baseline Scr (mg/dL) [IQR] & $0.31(0.23-0.46)$ & $0.35(0.24-0.52)$ & $0.29(0.23-0.47)$ \\
\hline Central access, n (\%) & $87(98.9)$ & $44(100)$ & $25(96.2)$ \\
\hline \multicolumn{4}{|l|}{ Goal vancomycin trough, $\mathrm{n}(\%)$} \\
\hline $10-20 \mathrm{mcg} / \mathrm{mL}$ & $78(88.6)$ & $39(88.6)$ & $25(96.2)$ \\
\hline $15-20 \mathrm{mcg} / \mathrm{mL}$ & $10(11.4)$ & $5(11.4)$ & $1(3.8)$ \\
\hline Positive cultures, n (\%) & $37(42)$ & $24(54.5)$ & $11(42.3)$ \\
\hline Vancomycin susceptible, n (\%) & $29(78.4)$ & $22(91.7)$ & $7(63.6)$ \\
\hline \multirow{7}{*}{ Diagnosis, n (\%) } & ALL-49 (56) & Bone- 9 (20) & Solid Tumor-19 (73) \\
\hline & AML-19 (21) & CNS- 8 (18) & Heme-7 (27) \\
\hline & Lymphoma-15 (17) & Neuroblastoma-6 (14) & \\
\hline & \multirow{4}{*}{ Other-5 (6) } & Rhabdomyosarcoma-6 (14) & \\
\hline & & Germ Cell-6 (14) & \\
\hline & & Wilms Tumor-3 (7) & \\
\hline & & Other-6 (14) & \\
\hline
\end{tabular}

Table 1: Patient demographics.

\begin{tabular}{|l|l|l|l|}
\hline \multirow{2}{*}{ Age (years) } & Heme & Solid Tumor & HSCT \\
\cline { 2 - 4 } & $\mathbf{( n = 7 6 )}$ & $\mathbf{( n = 3 6 )}$ & $\mathbf{( n = 2 4 )}$ \\
\hline$<1$ & $72.1[ \pm 6.7]$ & $90.0[ \pm 19.1]$ & $89.3[ \pm 8.6]$ \\
\hline $1-5$ & $83.6[ \pm 3.5]$ & $73.2[ \pm 4.9]$ & $74.2[ \pm 5.0]$ \\
\hline $6-11$ & $64.6[ \pm 3.9]$ & $74.4[ \pm 7.2]$ & $78.6[ \pm 6.7]$ \\
\hline$>11$ & $66.0[ \pm 5.1]$ & $52.7[ \pm 5.3]$ & $75.1[ \pm 5.6]$ \\
\hline$p$ value & $<0.05$ & $<0.05$ & 0.49 \\
\hline
\end{tabular}

Table 2: Mean dosage requirements by age $(\mathrm{mg} / \mathrm{kg} /$ day $)[ \pm \mathrm{SD}]$.

\begin{tabular}{|c|c|c|c|c|}
\hline \multirow[t]{2}{*}{ Parameter } & Heme & Solid Tumor & HSCT & \multirow{2}{*}{ p value } \\
\hline & $(n=88)$ & $(n=44)$ & $(n=26)$ & \\
\hline Mean difference in starting and therapeutic dosage $(\mathrm{mg} / \mathrm{kg} /$ day $)[ \pm \mathrm{SD}]$ & $14.2[ \pm 19.6]$ & $12.3[ \pm 17.6]$ & $14.1[ \pm 16.0]$ & 0.87 \\
\hline Attainment of goal, $\mathrm{n}(\%)$ & $75(85.2)$ & $36(81.8)$ & $24(92.3)$ & 0.5 \\
\hline Mean number of dose changes $[ \pm \mathrm{SD}]$ & $1.0[ \pm 0.1]$ & $1.2[ \pm 0.2]$ & $0.9[ \pm 0.2]$ & 0.7 \\
\hline Mean days to therapeutic $[ \pm$ SD] & $3.0[ \pm 0.2]$ & $3.3[ \pm 0.2]$ & $2.9[ \pm 0.3]$ & 0.6 \\
\hline Mean days of treatment $[ \pm S D]$ & $8.1[ \pm 0.7]$ & $6.7[ \pm 1.0]$ & $8.2[ \pm 1.3]$ & 0.5 \\
\hline
\end{tabular}

Table 3: Secondary outcomes. 


\section{Discussion}

It has been previously reported that vancomycin dosing requirements are increased in children with malignancy when compared to children that do not have malignancy [5]. Prior to this study there was no published literature evaluating differences in these requirements based upon category of malignancy in pediatric patients. One pharmacokinetic model of 348 courses of vancomycin therapy in 274 adult patients with hematologic malignancy or recent hematopoietic stem cell transplant (HSCT), reported a diagnosis of acute myeloid leukemia (AML) as a co-variant associated with increased clearance and volume of distribution [8]. As vancomycin dosage requirements are more variable among pediatric populations, further study into the impact of malignancy category on vancomycin dosage requirements in this population was deemed necessary.

The results of this study show that dosing requirements for vancomycin are not significantly different between children with hematologic malignancies, solid tumors, and those receiving HSCT. The average dose requirement found among those reaching therapeutic trough levels was $72.5[ \pm 2.3] \mathrm{mg} / \mathrm{kg} /$ day for hematologic malignancy, $66.5[ \pm 3.3] \mathrm{mg} / \mathrm{kg} /$ day for solid tumor, and $77.3[ \pm 4.1] \mathrm{mg} / \mathrm{kg} / \mathrm{day}$ for HSCT patients respectively. Within our hematologic malignancy group, 15 patients had a diagnosis of AML, with an average dosage requirement of $65.9 \mathrm{mg} / \mathrm{kg} /$ day. All of these averages are higher than the recommended vancomycin dosage of $60 \mathrm{mg} / \mathrm{kg} /$ day to treating MRSA infections, however not as high as the 80 to $90 \mathrm{mg} / \mathrm{kg} / \mathrm{day}$ reported in previous literature evaluating children with malignancy receiving vancomycin [6,7].

While patients with solid tumors had a lower dosage requirement, the increased incidence of supratherapeutic trough values demonstrated may warrant less aggressive dosing within this population. One potential confounder to this finding is the presence of central catheters in the majority of these patients, potentially resulting in trough samples contaminated with drug. This is supported by the observation that increased incidence of nephrotoxicity was not observed in this population. The HSCT population had the highest average dosage requirement, as well as an increased incidence of supratherapeutic trough values and nephrotoxicity. This outcome highlights the concerns with aggressively dosing patient's concurrent receiving nephrotoxic agents.

One factor not directly evaluated within this study was the dosing interval used. Intuitively patients within the $<1$ year and $1-5$ year age groups most frequently used the every 6 hour interval while patients in the 6-11 year and $>11$ year age group most frequently used the every 8 hour interval. Intervals used were similar between groups based on category of malignancy. Another consideration is the arbitrary therapeutic goal of $10-20 \mathrm{mcg} / \mathrm{mL}$ used for all patients in our statistical analysis, while a small number of courses (10\%) were actually targeting a vancomycin trough of 15 to $20 \mathrm{mcg} / \mathrm{mL}$ based upon site of infection.

Limitations to consider when evaluating the results of this trial include its single-center retrospective design. Although there were no significant differences found in baseline characteristics of the participants in this trial, patients were not matched between groups.
The prescribing practices of the ordering provider dosing vancomycin therapy could serve as a potential confounder. While a pharmacist prospectively reviewed all patients included in the study, $6 \%$ of the vancomycin courses evaluated were directed by a formal pharmacy consult service allowing the pharmacist to independently manage vancomycin therapy through a physician approved protocol. Additionally, while any patient with an estimated creatinine clearance under $50 \mathrm{~mL} / \mathrm{min} / 1.73 \mathrm{~m}^{2}$ was excluded from the study, there may have been renal function changes during patient's therapy that contributed to confounding of their true dosage requirement. As patients were commonly on concomitant nephrotoxins throughout the study, it is not possible to tell whether the nephrotoxicity noted was a direct result of the vancomycin therapy. Patients were also assumed to have achieved steady state concentrations of vancomycin after the administration of at least two doses of the drug.

\section{Conclusion}

In conclusion while vancomycin dosage requirements are similar between pediatric patients with differing categories of malignancies, there appear to be differences in their susceptibility to toxicity with similar dosage regimens. Patients with solid tumors appear to be at higher risk for supratherapeutic trough values at lower dosages when compared to patients with hematologic malignancies and those who have undergone HSCT. Patients status post HSCT also appear to be at an increased risk for supratherapeutic trough values and also for nephrotoxicity, having received the highest average doses of vancomycin and most concomitant nephrotoxins. These findings should be taken into consideration when dosing vancomycin in children with malignancy to avoid nephrotoxicity and supratherapeutic trough values. Further study is required to determine how this risk for toxicity impacts clinical practice.

\section{Acknowledgment}

The findings of this study were presented on April 282016 at the Pediatric Pharmacy Advocacy Group Annual Meeting in Atlanta, Georgia. The authors of this study would like to acknowledge Stacy Voils, PharmD, M.S., BCPS for providing statistical support and Benjamin Staley, PharmD, BCPCS for his assistance in data retrieval for this research.

\section{Disclosure}

The author(s) declare no conflicts or financial interest in any product or service mentioned in the manuscript, including grants, equipment, medications, employment, gifts, and honoraria. The primary author, Kyle Franco, PharmD, had full access to all the data in the study and takes responsibility for the integrity of the data and the accuracy of the data analysis.

\section{References}

1. Lehrnbecher T, Phillips R, Alexander S, Alvaro F, Carlesse F, et al. (2012) Guideline for the management of fever and neutropenia in children with 
Citation: Franco KA, Higgins T (2017) Impact of Malignancy Category on Vancomycin Dosage Requirements in Pediatric Oncology Patients. J Pharmacovigil 5: 238. doi:10.4172/2329-6887.1000238

Page 5 of 5

cancer and/or undergoing hematopoietic stem-cell transplantation. J Clin Oncol 30: 4427-4438.

2. Liu C, Bayer A, Cosgrove, Daum RS, Fridkin SK, et al. (2011) Clinical practice guidelines by the infectious disease society of America for the treatment of methicillin-resistant Staphylococcus aureus infectious in adults and children. Clin Infect Dis 52: e18-e55.

3. Freifeld AG, Bow EJ, Sepkowitz KA, Boeckh MJ, Ito JI, et al. (2011) Clinical practice guideline for use of antimicrobial agents in neutropenic patients with cancer: 2010 update by the infectious disease society of America. Clin Infect Dis 52: e56-e93.

4. Le J, Bradley JS, Murray W, Romanowski GL, Tran TT, et al. (2013) Improved vancomycin dosing in children using area under the curve exposure. Pediatr Infect Dis J 32: e155-e163.

5. Krivoy N, Peleg S, Postovsky S, Ben Arush MW (1998) Pharmacokinetic analysis of vancomycin in steady state in pediatric cancer patients. Pediatr Hematol Oncol 15: 333-338.
6. Zhao W, Zhang D, Fakhoury M, Fahd M, Duquesne F, et al. (2014) Population pharmacokinetics and dosing optimization of vancomycin in children with malignant hematological disease. Antimicrob Agents Chemother 58: 3191-3199.

7. Sanders SJ, Bijleveld YA, Sinkeler F, Kemper EM, Pajkrt D, et al. (2014) Clinical evaluation of vancomycin dosage in pediatric oncology patients. Pediatr Infect Dis J 33: 731-733.

8. Buelga DS, Del mar fernandez de gatta M, Herrera EV, Dominguez-Gil A, Garcia MJ (2005) Population pharmacokinetic analysis of vancomycin in patients with hematological malignancies. Antimicrob Agents Chemother 49: 4934-4941. 